\title{
An Automated System for Prediction of Icing on the Road
}

\author{
Konstantin Korotenko \\ P.P. Shirshov Institute of Oceanology \\ 36 Nakhimovsky pr. Moscow, 117851, Russia \\ http://www.aha.ru/ koroten \\ koroten@aha.ru
}

\begin{abstract}
During the period from late autumn to early spring, vast areas in North America, Western Europe, and many other countries experience frequent snow, sleet, ice, and frost. Such adverse weather conditions lead to dangerous driving conditions with consequential effects on road transportation in these areas. A numerical forecasting system is developed for automatic prediction of slippery road conditions at road station sites in northern Europe and North America. The system is based on a road conditions model forced by input from an operational atmospheric limited area model. Synoptic information on cloud cover and observations of temperature, humidity, water and ice on the road from the road station sites are taken into account in a sophisticated initialization procedure involving flux corrections for individual locations. The system is run initially at the Rhode Island University with promising results. Currently, new forecasts $3 \mathrm{~h}$ ahead are produced every 20 minutes for 14 road station sites.
\end{abstract}

\section{Introduction}

An accurate prediction of meteorological parameters such as precipitation, temperature, and humidity close to the ground is of great importance for various applications. For example, warnings about slippery road conditions may be issued if snow, freezing rain, or rime can be forecast with sufficient accuracy. An addition, traffic delays and the risk of accidents may be significantly reduced by specific actions such as road salting. An impressive amount of money is spent on winter road maintenance in many European countries. For example, it is estimated that the total budget for winter road maintenance in the United Kingdom is about $\$ 200$ million every year. For Denmark, being a smaller country, the corresponding budget is about half of this. The variability from year to year, however, is considerable. Unnecessary road salting should be avoided for economic reasons and due to the risk of environmental damage. This means that optimal salting procedures should be sought. In this context, accurate road weather information is vital, which justifies the efforts that are spent on the development of advanced road conditions models. The present paper concerns the development of a numerical model system for operational forecasting of the road conditions in Rhode Island, USA. The prediction of the road conditions requires the production of accurate forecasts of temperature, humidity, and precipitation at the 
duction of accurate forecasts of temperature, humidity, and precipitation at the road surface. To provide this information, two strategies are possible. The first one relies on the manual work of a forecaster who issues warnings of slippery road conditions based on various meteorological tools, for example, synoptic observations and output from atmospheric models. The second possibility is based on automatic output from specialized models, which may involve statistical or deterministic methods. The two approaches can be used in combination if a forecaster supplies certain input data for the automatic system

\section{System Basic Formulae and Boundary Conditions}

The system is primarily based on earlier models developed by Sass [6] and Baker and Davies [1]. Additional work by Unsworth and Monteith [8], Strub and Powell [7], Louis [4], Jacobs [2] and Manton [5] provided information needed in the parameterization of the atmospheric heat flux terms. The resulting second order diffusion equation, with empirically parameterized flux terms, is solved by a standard forward in time, centered in space finite difference scheme. The model predicts the continuous vertical temperature profile from the road surface to depths of about two meters in the roadbed. The model also allows predictions of road icing conditions. Atmospheric data, necessary as input to the model, can be supplied either from observations or from a weather forecast model.

\subsection{Ground Heat Flux}

The model is constructed on an unsteady one-dimensional heat conduction equation, that is,

$$
\frac{\partial T_{S}}{\partial t}=\frac{\lambda_{G}}{\rho_{G} C_{G}} \frac{\partial^{2} T_{S}}{\partial t^{2}},
$$

where $T_{s}(z . t)$ is the temperature at time $t$ and depth $z$. It is assumed that the road surface and underlying sublayers are horizontally homogeneous so that heat Transfer in horizontal direction can be neglected- The model considers a vertical pillar with unit cross-sectional area, extending to the depth (usually $2 \mathrm{~m}$ ) deep enough to eliminate the diurnal oscillation of temperature. The equation is solved with finite-difference method [3], along with an initial temperature profile within the road sublayer and upper and lower boundary conditions.

The initial condition prescribes the temperature at every grid point in the road sublayers at the beginning of forecast. The lower boundary condition is straightforward and treated as a constant of mean winter soil temperature at two meters. The upper boundary condition is complicated and is expressed by an energy balance equation. 
The grid spacing is irregular with thin layers close to the surface. The temperature at the bottom layer is determined by a climatological estimate depending on the time of year. The values of the heat conductivity $\lambda_{G}$, density $\rho_{G}$, and specific heat capacity $C_{G}$ are constant (see Apendix).

\subsection{Solar Heat Flux}

The solar and infrared radiation is computed from the radiation scheme used in the atmospheric HIRLAM model [6]. The net solar flux density $\phi_{R s}$ at the ground is computed according to (2) as a linear combination of a clear air part $\phi_{R s a}$ and a cloudy part $\phi_{R s c}$.

$$
\phi_{R s}=(1-\alpha)\left[\phi_{R s a}\left(1-C_{M}\right)+\phi_{R s} C_{M}\right] \text {, }
$$

where $\alpha$ is a surface albedo for solar radiation and $C_{M}$ is a total cloud cover determined from a maximum overlap assumption. The clear air term is parameterized in (3):

$$
\begin{array}{r}
\phi_{R s a}=S \cos \theta\left(1-0.024 \cos \theta^{-0.5}-a_{6} 0.011 u_{s}^{0.25}\right) \\
-S \cos \theta \frac{p}{p_{00}} a_{7}\left[\frac{0.28}{1+6.43 \cos \theta}-0.07 \alpha\right]
\end{array}
$$

where $S$ is the solar constant and $p_{00}$ is a reference pressure. The first term in (2) depending on the zenith angle concerns the stratospheric absorption due to ozone. A major contribution to the extinction of solar radiation comes from tropospheric absorption due to water vapor, $\mathrm{CO}_{2}$, and $\mathrm{O}_{3}$. This is parameterized according to the second term in (3). Here, $U_{S}$ is the vertically integrated water vapor path throughout the atmosphere. It is linearly scaled by pressure and divided by $\cos \theta$. The last term involving two contributions describes the effect of scattering. The first contribution arises from scattering of the incoming solar beam, while the second one is a compensating effect due to reflected radiation, which is backscattered from the atmosphere above. The coefficients $a_{6}$ and $a_{7}$ (see appendix) that are larger than 1 represent a crude inclusion of effects due to aerosol absorption and scattering, respectively.

The cloudy contribution $\phi_{R s c}$ of (2) is given by (4):

$$
\phi_{R s c}=\phi_{R s H} \frac{\hat{T}\left(p_{H}, p_{s}\right)}{1-\alpha\left[1-\hat{T}\left(p_{H}, p_{s}\right)\right] a_{8}} .
$$

In (4), $\phi_{R s H}$ is the solar flux density at the top of the uppermost cloud layer. It is given by a formula corresponding to (3), and the surface albedo appearing in the back- 
scattering term of (3) is replaced by an albedo representing the cloudy atmosphere below. The transmittance of flux density from top to bottom of the cloudy atmosphere is described by $\hat{T}\left(p_{H}, p_{s}\right)$. In (4), the denominator takes into account multiple reflections between ground and cloud. The constant ay accounts for absorption in reflected beams.

\subsection{Longwave Radiative Heat Flux}

The outcome of the radiation computations is the net radiation flux $\phi_{R}$, which can be partitioned into shortwave and longwave contributions:

$$
\phi_{R}=R_{S}[l-\alpha(\theta, F)]+\varepsilon_{S}\left(R_{L}-\sigma T_{S}^{4}\right),
$$

where $\alpha(\theta, F)=\alpha_{1}(\theta)+\left[C_{1}-\alpha_{1}(\theta)\right] F, \quad F=\min \left(H / H_{C}, 1\right)$,

$$
\alpha_{1}(\theta)=\left\{\begin{array}{l}
\alpha_{0} \text { if } \cos (\theta) \geq C_{3} ; \\
C_{2}+\alpha_{0}\left(1-C_{2}\right)-\left(C_{2} / C_{3}\right) \cos (\theta)\left(1-\alpha_{0}\right) \text { if } \cos (\theta)<C_{3}
\end{array}\right. \text {, }
$$

and

$$
C_{1}=0.60, C_{2}=0.10, C_{3}=0.60 ; \alpha_{0}=0.10, H_{C}=0.0010
$$

In (5), $\alpha$ is the road-surface shortwave albedo, $F$ is a scaled, dimensionless icesnow height, and $H$ is ice-snow height (m) of equivalent water. There is no distinction between ice and snow. In addition, $H e$ is a critical value for ice-snow height, $\theta$ is the solar zenith angle, and $R_{S}$ is the total downward solar flux consisting of direct and diffuse radiation. Effects of absorption by water vapor and clouds are included, as is scattering of radiation by clear air and clouds.

The value $R_{L}$ is the infrared longwave flux reaching the road surface, which absorbs only a fraction $\varepsilon_{S}=0.90$. It consists of longwave radiation from clear air and clouds, which have an emissivity less than 1, depending on cloud type and thickness. The emissivity of clouds in the lower part of the atmosphere is, however, close to 1 , provided that the clouds are sufficiently thick $(\sim 200 \mathrm{~m})$. The upward emission of longwave radiation is $\mathcal{E} \sigma T_{S}^{4}$, where $\sigma$ is the Stefan-Boltzmann constant, $\sigma=5.7^{*}$ $10^{8} \mathrm{Wm}^{-2} \mathrm{~K}^{-4}$, and $T_{s}$ is the road surface temperature $(K)$.

According to observations [6], the albedo $a_{l}(\theta)$ for natural surfaces increases for large solar zenith angles, but it is almost constant equal to $\alpha_{0}=0.60$ for $\cos (\theta)>$ 03 , as expressed in (1). For most surfaces, $0<\alpha_{0}<0.30$, and for asphalt roads it is reasonable to assume that $\alpha_{0}=0.10$. For simplicity, the albedo for large zenith angles has been expressed as a linear function of $\cos (\theta)$. This involves the introduction 
of an additional constant $C_{2}$. The value of $C_{2}$, however, is not well known and should ideally be based on local measurements. The constant $c_{1}=0.60$ represents a common albedo for ice and snow, which may have an albedo in the range between 0.35 for slushy snow and 0.80 for fresh snow [6]. Because of this simplification, the zenith-angle dependency of albedo is neglected in case of ice or snow. In order to prevent a discontinuous transition between no ice and ice conditions, a simple interpolation term involving the dimension less ice height $F$ is added to a $a_{1}(\theta)$ to obtain the albedo $a(\theta, F)$.

\subsection{Longwave Radiative Heat Flux}

Traditional drag formulas as given by (6) and (7) are used to describe the fluxes of sensible and latent heat:

$$
\begin{aligned}
\phi_{S} & =C_{p} \rho_{z} C_{S}\left|V_{z}\right|\left(\theta_{z}-\theta_{s}\right), \\
\phi_{Q} & =L_{v s} \rho_{z} C_{Q}\left|V_{z}\right|\left(q_{z}-q_{s}\right), \\
C_{S} & =C_{q}=\left[\frac{k}{\ln (Z / z)}\right]^{2} f\left(R i, \frac{Z}{z_{0}}\right), \text { and } \\
q_{S} & =\left(\frac{W_{s}}{W_{c}}\right) q_{s a t}\left(T_{S}\right)+\left(1-\frac{W_{s}}{W_{c}}\right) q_{z},
\end{aligned}
$$

where

where $\mathrm{Z}=10 \mathrm{~m}, C_{p}$ is the specific heat of moist air at constant pressure, $p_{z}$ is the air density, and $\theta_{z}$ and $\theta_{s}$, are potential temperatures at the computation level $\mathrm{Z}$ and at the surface, respectively. Similarly, $q_{z}$ and $q_{s}$ are specific humidities at the same levels. The latter is determined by a surface wetness parameter $W_{s} W_{c}$, where $W_{c}=0.5 \mathrm{~kg}$ $\mathrm{m}^{-}{ }^{2}\left(0<W_{s} / W_{c}<1\right), q_{s a t}\left(T_{s}\right)$ is the saturation specific humidity at the surface temperature $T_{s}$, and $L_{v s}$ is the specific latent heat of evaporation if $T_{s}>0^{\circ} \mathrm{C}$, otherwise it is the specific heat of sublimation. Here. $k$ is the von Karman constant.

\section{Description of the Developed System}

The system was developed with a use of Visual Basic 6.0, Compaq Digital Fortran 6.0 , ArcView GIS, and Surfer 7.3. The system is currently operational, in rudimentary form. The development of the web-based interface was being coordinated with a similar effort funded by the EPA EMPACT program and led by the Narragansett Bay Commission. The system allows access to a base map, GIS data on primary and sec- 
ondary highways from RI GIS, and linkages to a variety of supporting web sites. As an example Figure 1 shows the opening page of the web site. It displays a map of RI and shows the location of the RWIS observation site.

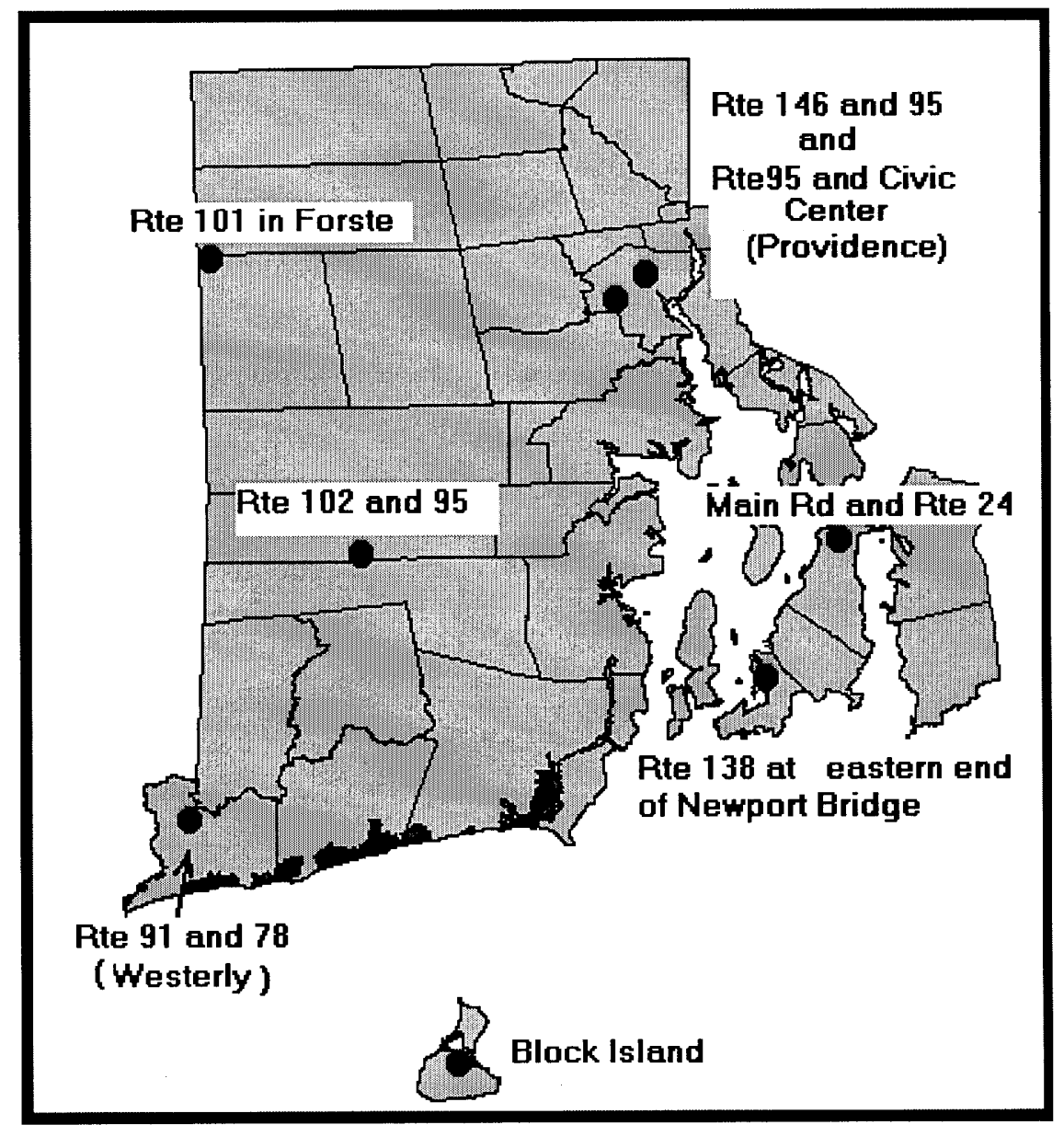

Fig. 1. Rhode Island State (USA) map. The Road Weather Information System (RI RWIS) sites are depicted by solid circles 


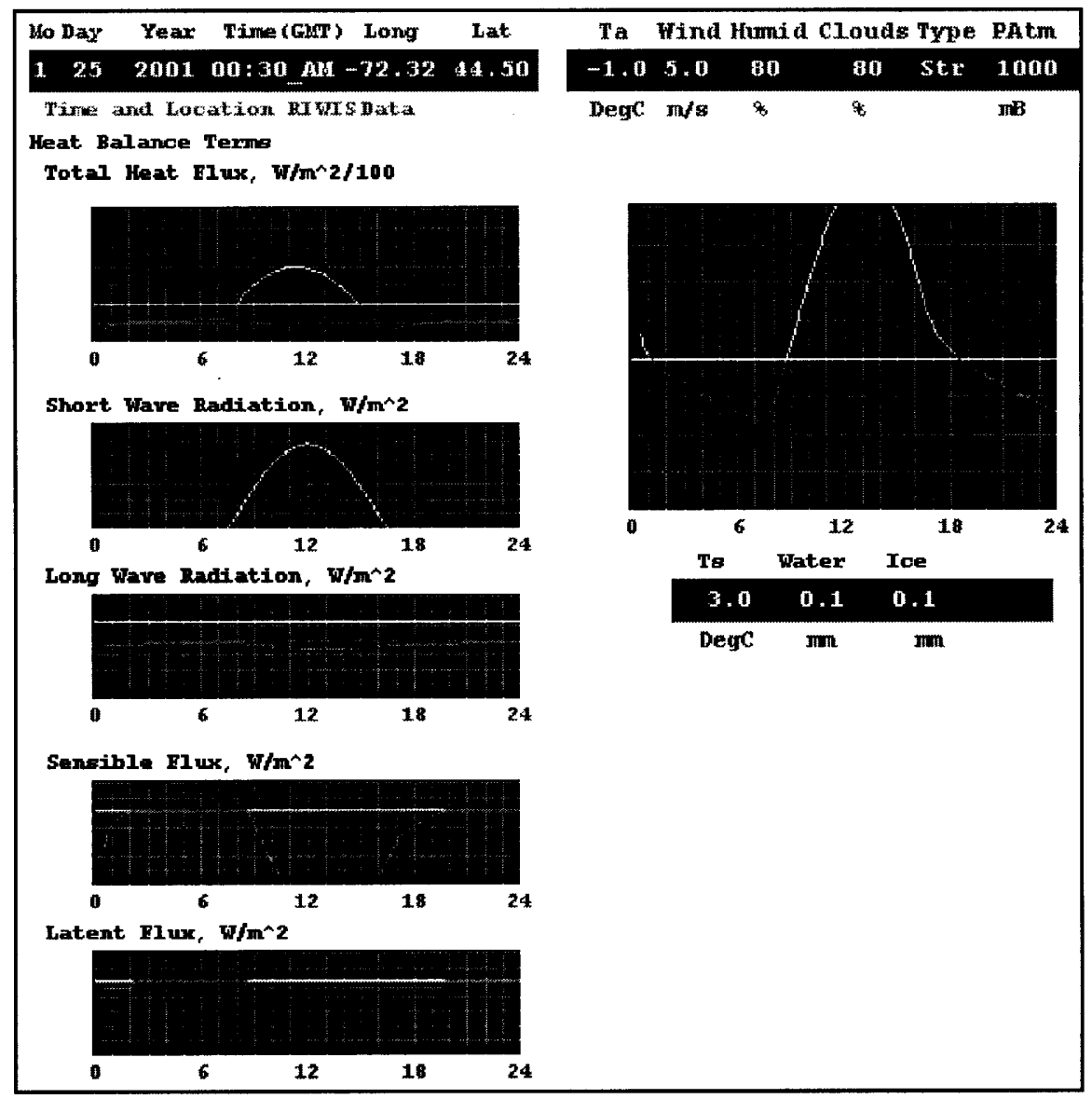

Fig. 2. Heat balance terms and the road temperature predicted for the site $72.32 \mathrm{~W}, 44.50 \mathrm{~N}$

As an example Figure 2 illustrate the heat balance terms and the road temperature calculated on January 25,2001 at the location $72.32 \mathrm{~W}, 44.50 \mathrm{~N}$. It is seen that changes of the total heat leads to the oscillation of the pavement temperature that leads, in turn, to the formation rime and slippery roads in the evening and night, and wet or dry roads at daytime.

Acknowledgements. Author wishes to thank M. Spaulding, C. Calagan and T. Opishinski for fruitful discussion and support of this work . 


\section{References}

1. Barker, H.W. Davies, J.A.: Formation of Ice on Roads beneath Bridges. Journal of Applied Meteorology, Vol.29, (1990) 1180-1184.

2. Jacobson, M.Z., Fundamentals of Atmospheric Modeling. Cambridge University Press (1999) $656 \mathrm{p}$.

3. Korotenko, K.A.: Modeling Turbulent Transport of Matter in the Ocean Surface Layer. Oceanology, Vol.32, (1992) 5-13.

4. Louis, J. F.: Parameteric Model of Vertical Eddy Fluxes in the Atmosphere. Boundary Layer Meteorology, Vol. 17, (1979) 187-202.

5. Manton, M. J.: A Bulk Model of the Well-Mixed Boundary Layer, Boundary-Layer Meteorology, Vol.40, (1987) 165-178.

6. Saas, B. H.: A Numerical Forecasting System for the Prediction of Slippery Roads. Journal of Applied Meteorology, Vol. 36 (1996) 801-817.

7. Strub, P.T., Powell, T.M.: The Exchange Coefficients for Latent and Sensible Heat Flux over Lakes: Dependence Upon Atmospheric Stability. Boundary-Layer Meteorology, Vol. 40 (1987) 349-361.

8. Unsworth M.H., Monteith, J.L.: Long-Wave Radiation at the Ground. I. Angular Distribution of Incoming Radiation. Quart. J. R .Met. Soc., Vol. 101 (1975) 13-24.

\section{Appendix: Model Coefficients}

Table 1. Model Coefficients

\begin{tabular}{llllll}
\hline Coefficient & Value & Coefficient & Value & Coefficient & Value \\
\hline$a_{1}$ & $5.56^{*} 10^{-5}$ & $B_{1}$ & 35 & $k$ & 0.40 \\
$a_{2}$ & $3.47^{*} 10^{-5}$ & $B_{2}$ & 3000 & $L_{s}$ & $2.83^{*} 10^{6}$ \\
$a_{3}$ & 0.25 & $B_{3}$ & 0.60 & $L_{v}$ & $2.50^{*} 10^{6}$ \\
$a_{4}$ & 600 & $B_{4}$ & 0.17 & $W_{s}$ & 0.5 \\
$a_{5}$ & $2.78^{*} 10^{-5}$ & $B_{5}$ & 0.0082 & $\alpha$ & 0.10 \\
$a_{6}$ & 1.20 & $B_{6}$ & 0.0045 & $\varepsilon_{0}$ & 0.90 \\
$a_{7}$ & 1.25 & $B_{7}$ & 0.4343 & $\lambda_{G}$ & 2.0 \\
$a_{8}$ & 0.80 & $B_{8}$ & $2.5^{*} 10^{3}$ & $\rho_{G}$ & 2400 \\
$a_{9}$ & 20 & $C_{G}$ & 800 & $\sigma$ & $5.67^{*} 10^{-8}$ \\
$a_{10}$ & 40 & $D_{o o}$ & $5^{*} 10^{4}$ & & \\
$a_{11}$ & 1 & $G$ & 9.81 & & \\
\hline
\end{tabular}

